DOI: $10.1007 / \mathrm{s} 13367-011-0022-6$

\title{
Analysis of drag effects on pulsatile blood flow in a right coronary artery by using Eulerian multiphase model
}

\section{Fuat Yilmaz, Ahmet Ihsan Kutlar and Mehmet Yasar Gundogdu*}

University of Gaziantep, Faculty of Engineering, Department of Mechanical Engineering, 27310, Gaziantep/Turkey

"Analysis of drag effects on pulsatile blood flow in a right coronary artery by using Eulerian multiphase model”. Fuat Yilmaz, Ahmet Ihsan Kutlar and Mehmet Yasar Gundogdu*. Vol. 23, No. 2 (2011) pp. 89103.

Equation 12 on page 91 and Equation 27 on page 94 should be corrected as follows:

$$
\begin{aligned}
\mathrm{F}(\alpha) & =\mathrm{e}^{\mathrm{k}_{1} \alpha}+\alpha^{\mathrm{k}_{2}} \\
\eta_{\mathrm{r}} & =\frac{\mu_{\text {mix }}}{\mu_{\text {plasma }}}=\frac{\mu_{\mathrm{RBC}} \alpha_{\mathrm{RBC}}+\alpha_{\text {plasma }} \mu_{\text {plasma }}}{\mu_{\text {plasma }}}
\end{aligned}
$$

